\title{
Early infant diagnosis of HIV in India-Early results and sero-positivity determinants
}

\author{
Urvish Joshi ${ }^{1 *}$, Umesh Oza ${ }^{2}$, Anand Solanki ${ }^{1}$, Sheetal Vyas ${ }^{1}$, Heena Modi ${ }^{2}$ \\ ${ }^{1}$ Department of Community Medicine, AMC MET Medical College, Ahmedabad, India; \\ *Corresponding Author: doc.urvish@gmail.com \\ ${ }^{2}$ Ahmedabad Municipal Corporation AIDS Control Society, Ahmedabad, India
}

Received 10 June 2012; revised 16 July 2012; accepted 9 August 2012

\section{ABSTRACT}

Introduction: PMTCT under NACP-III cover exposed children born to sero-positive mothers. Baby's sero-status could be confirmed only at 18 months. Under EID, by DBS and/or WB collection, DNA-PCR can be performed earlier, with subsequent ART-linkage and 18-months-confirmation. In Ahmedabad, with 55,000 annual pregnancy-HIV-testing, sero-prevalence is $0.27 \%$. Methodology: Entry-points in EID are at 6 weeks, 6 months or 12 months. Cohort of 213 exposed children since EID roll-out (June 2010-December 2011) at all tertiary care hospitals under Ahmedabad Municipal Corporation was assessed for sero-positivity-prevalence, DBS validity and assessment of baby's sero-status-determinants. De-identified, secondary data were captured under routine public-health-program. Necessary permissions taken. Results: 144 HIV sero-positive deliveries took place. 213 exposed children were enrolled in EID. Cumulatively, 18 (8.45\%) were tested positive at all entry-points. Out of sero-positives confirmed at 18 months, $60 \%$ children's mothers were detected either in second or third trimester. In $40 \%$, mothers remained undiagnosed intra-partum. Mothers were not on ART intra-partum in $\mathbf{8 0 \%}$ (RR 1.8). Peri-partum ARV prophylaxis-single-dose-Nevirapine (sdNVP) was not given in $60 \%$. [RR $18, \mathrm{Cl} 3.69$ to 87.70 at $95 \%(p<0.0003)]$. In $60 \%$, mode of delivery was vaginal, deliveries were handled in emergency. History of exclusive breastfeeding was in $60 \%$. Discussion: Rise in yield of sero-positivity with age, highest proportion of sero-positivity and highest number of entrants at 6 weeks call for efforts targeted towards increasing earliest EID uptake clubbed with immunization visits. Feasibility, validity and early-ART-linkage to reduce mortality are features of DBS. Results justify its use in national program. Earliest pregnancy-HIV detection, HIV-testing for emergency deliveries, intra-partum sdNVP to both mother and baby, ART-linkage of eligible mothers and following infant feeding guidelines remain cornerstone of PMTCT success.

Keywords: PMTCT; Early-Infant-Diagnosis (EID); Dried-Blood-Spot (DBS); HIV Sero-Positivity Determinants; Vertical Transmission

\section{INTRODUCTION}

Effective health care delivery to the majority of perinatally exposed infants worldwide, including those enrolled in prevention of mother-to-child transmission (PMTCT) programs, is hampered by lack of access to an HIV diagnosis in infancy.

Operationally, such children born to sero-positive mothers are exposed children and are beneficiaries of the prevention-from-parent-to-child-transmission (PPTCT) services provided under National AIDS Control Program-III (NACP-III) and are followed at government health facilities. Diagnosis of HIV status in these children is very important in order to plan their care, support and treatment.

According to earlier PMTCT program in India, the only diagnosis available for exposed children was by rapid tests at the age of 18 months and the future ART linkage would depend upon that. Presence of maternal antibodies against HIV in the child's blood was the reason for delayed diagnosis. A crucial period in terms of therapy, continuous care and support would be missed in many such cases.

A more accurate option for them is antigen-specific DNA polymerase chain reaction (DNA PCR) test that detects HIV-1 pro-viral DNA integrated to human genome [1-3] which has been implemented across India from 2010.

The test specimen for DNA-PCR can be collected in 
two ways: Dried Blood Spot (DBS) and Whole Blood (WB). Out of these, WBS requires sophisticated, expensive equipment, and the samples have to be sent to the laboratory within 24 hours under proper cold chain maintenance, while DBS can be stored and shipped to testing facilities without refrigeration within 15 days [4]. Thus DBS collection from young infants for centralized HIV-PCR testing is attainable in low-resource settings [1]. National guideline under Early Infant Diagnosis of HIV (EID) is to use DBS as a first test and WBS as the confirmatory test if the child is found positive from DBS. DBS, at the earliest, is advised at 6 weeks of age i.e. for the infants below 6 months of age, while for children above 6 months of age, a combination of rapid test and subsequent DBS is advocated if the former turns out positive [5].

Ahmedabad is one of the metro cities of India having more than 6 million of population and about 60,000 of pregnant women tested annually for HIV. The seroprevalence of HIV remains to be $0.22 \%$ among the tested women [6].

It has been more than one and half years since implementation of EID in Ahmedabad and it calls for the need to assess the validity of the earliest diagnosis of exposed child through DBS in the field.

A cohort of 213 exposed children at four major tertiary care hospitals under Ahmedabad Municipal Corporation (AMC) area was assessed for prevalence of sero-positivity, validity of DBS and assessment of determinants affecting the child's sero-status.

\section{METHODOLOGY}

\subsection{EID Protocol under NACP III}

The ICTC-PPTCT counselor coordinates the linkages and services required for care of exposed children. That includes follow-up of the exposed child as well as its algorithm-based testing under EID with DNA-PCR technique at the landmarks of 6 weeks, 6 months or 12 months and finally confirmatory testing is done at 18 months. Pre and post-test counseling is provided during each testing as per the client requirement (Figure 1).

\subsection{EID in AMC}

AMC-AIDS Control Society (AMC-ACS) is implementing EID under PPTCT services since May-June 2010 in its centers after training of concerned staff. DBS and WB samples collected from exposed infants are sent to the regionally-identified center-Kastoorba Hospital, Mumbai, for DNA-PCR testing.

\subsection{Study Population and Design}

Six weeks is the earliest age at which PCR is expected to detect virtually all perinatally transmitted HIV infection. National guidelines allow the earliest DBS collection at the same time. At the age of 18 months, confirmatory testing is to be made by three regular rapid tests. All children enrolled in EID under AMC since its rollout, regardless of their entry-point, their influx source and their current status, constituted the cohort for a retrospective prospective study design.

\subsection{Study Duration}

From June 2010 (EID inception) till December 2011.

\subsection{Ethical Issues}

Written informed consent was taken from the mother/ parent/guardian of the exposed child before each testing



Figure 1. Schedule of visits for HIV exposed infants and children $<18$ months. 
procedure and confidentiality was maintained throughout the time period by the counselor appointed under NACP.

The data analyzed here are the de-identified and secondary, captured under routine public health program. Permission for the same was taken from AMC-ACSthe city-chapter of National AIDS Control Organization (NACO).

\section{RESULTS}

Since the roll out of EID in June 2010, there were 144 HIV sero-positive deliveries till December 2011; total 213 exposed children were enrolled in EID system since the roll-out.

Cotrimoxazole Prophylaxis Therapy (CPT) was started in all exposed children tested positive at 6 weeks of age.

Out of total 213 exposed children at different entrypoints of EID, 18 were tested positive (8.45\%) while 195 turned out to be negative (91.55\%).

Entry-point-wise yield of sero-positivity in these exposed children under EID system was as per Figure 2. Sero-positivity was $16.7 \%$ among exposed children tested at 18 months while it was $5.2 \%$ at 6 weeks of testing.

Proportionate break-up of sero-status of all exposed children tested over various entry-points in EID was as per Figure 3. Proportion of sero-positivity at 6 weeks of testing was $38.9 \%$ while it was $5.6 \%$ at 18 months of confirmatory testing.

Main channels for the influx of exposed children in EID remain to be either routine PPTCT follow-up or direct referral from the pediatric units of the concerned healthcare setting. Break-up of exposed children under
EID according to their source of in-referral and their living status was as per Table 2.

26 Children tested at 6 weeks, reached landmark of confirmatory testing at 18 months. Out of them, 2 were confirmed sero-positive. Results at 6 weeks and 18 months testing were in agreement (Table 3).

Sensitivity and specificity of DBS testing at 6 weeks were $100 \%$. Similarly, positive and negative predictive values of the same were also $100 \%$.

Both these children were linked to ART services after DBS at 6 weeks.

Various other peri-partum factors were correlated with sero-status of the children undergoing confirmatory 18 months testing (Table 4).

$60 \%$ of all sero-positive children's mothers were detected either in second or third trimester. In $40 \%$ of the cases, mothers remained undiagnosed and were detected post delivery.

$80 \%$ of confirmed sero-positive children's mothers were not on ART at the time of delivery. Relative risk (RR) was 1.8 at $95 \%$ confidence interval (CI) of 0.2222 to 16.0226 (z statistic insignificant).

Ante-retroviral (ARV) prophylaxis in the form of single-dose-Nevirapine (sdNVP) as mother-baby-pair (MBPair) was not given in $60 \%$ of confirmed sero-positive children (RR 18 at 95\% CI of 3.6943 to 87.7022 (p < $0.0003)$, statistically highly significant).

In $60 \%$ of confirmed sero-positive children, mode of delivery was vaginal and the deliveries were handled in emergency (RR 0.79 and 0.89 respectively, z statistically insignificant) while history of exclusive breastfeeding was reported in $60 \%$ of confirmed sero-positive children

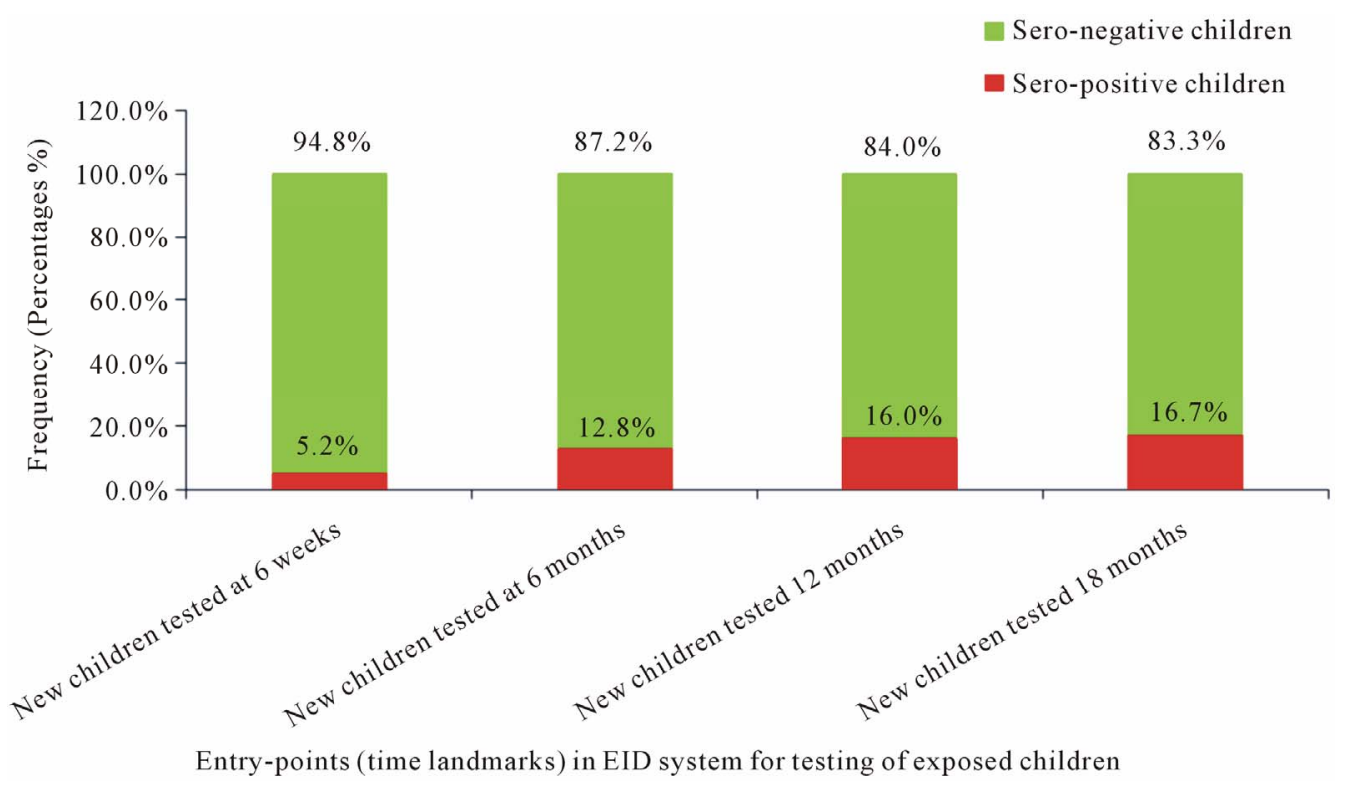

Figure 2. Yield of HIV sero-positivity at different entry-points of EID system. [All enrolled exposed children-inclusive of pediatric in-referral along with regular PPTCT follow-up, regardless of alive-status]. 


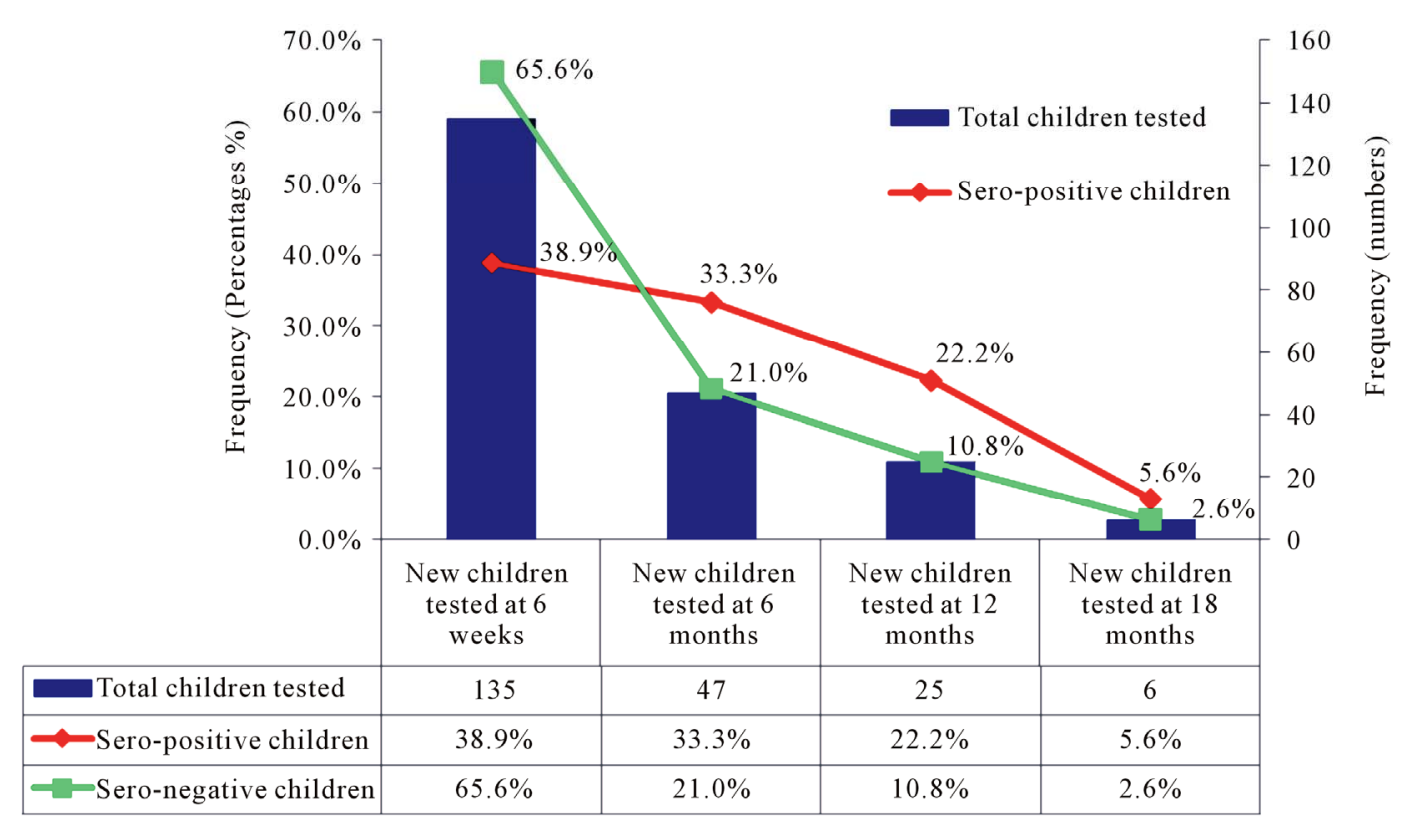

Figure 3. HIV sero-status proportions at different entry-points in EID system [all enrolled exposed children-inclusive of pediatric in-referral along with regular PPTCT follow-up, regardless of alive-status].

(RR 0.20, z statistically insignificant).

\section{DISCUSSION}

Since inception of EID in the Ahmedabad Municipal Corporation area, under PPTCT services, 144 HIV seropositive pregnant women delivered. Children born to seropositive mothers are termed as exposed children. Children enrolled for PPTCT services even before inception of EID and crossing the testing-entry-points under protocol thereafter were also added to the pool of total exposed children being tested. Such 213 children thus formed the pool of EID. There was a case of twin delivery as well and both children were enrolled in EID.

Yield percentages were calculated from the babies previously not tested and entering into the EID system at different entry-points i.e. 6 weeks, 6 months or 12 months, as well as for confirmatory testing at 18 months. The yield distribution showed that the percentages of detection of sero-positivity increased among the new entrants from testing at 6 weeks $(5.2 \%$-lowest $)$ to testing at 18 months (16.7\%-highest) (Figure 2). Rise in yield of sero-positivity among fresh entrants suggests the rise in the risk of contracting HIV as the age increases. Naturally, PPTCT services would not be utilized till the time baby is tested under EID. These services comprise of early detection of the baby and subsequent ART linkage, CPT services as well as proper counseling, guidance and follow-up on feeding and nutritional practices.

Out of all 213 children tested under PPTCT after June 2010, out of 213, 78 children could be tested at 18 months with confirmatory tests, out of which 5 turned out to be sero-positive (6.41\%). Our study took place in the Ahmedabad, the biggest city of the state of Gujarat. Jain et al. in their study found the sero-prevalence of $11.4 \%$ in 326 children across the state of Gujarat involving PPTCT centers of all tiers i.e. primary, secondary and tertiary [4]. Current study involved the PPTCT centers only in tertiary care hospitals having well-established referral and follow-up services since inception which might add to the decrease in sero-prevalence.

Highest proportion of sero-positivity was detected at 6 weeks of testing (38.9\%) which is the earliest; sero-status of a baby can be detected under the national program. Proportion of sero-positivity gradually declined with testing at 6 months and 12 months and was reduced to the lowest $5.6 \%$ at 18 months of confirmatory testing (Figure 3).

Besides, EID entry-point of 6 weeks saw the highest number of babies (135) coming in for testing compared to 18 months of confirmatory testing (6) (Table 1). The numbers steadily declined over the time. The EID system has been initiated in the municipal corporation area only about 18 months back and several babies born thereafter at various points of time may need some time to catch up with the confirmatory testing age of 18 months. Moreover, death of exposed children by the time they reach 18 months of age is also a worrisome issue. Crucial CD4 counts would drop in several undetected cases by 18 months and the ART response thereafter would not be as desired leading to subsequent mortality. In most resource-limited settings of India, drop-out rates for even routine immunization services are almost constant which also hamper the crucial follow-up of 18 months. 
Aforementioned factors collectively boost the importance of earliest detection of exposed child for HIV i.e. at 6 weeks, during the first major follow-up visit of the mother with her child to the healthcare facility. The main purpose of the same would be her post-natal check-up and child's immunization. Effective clubbing of EID at this point of time needs to be further stressed upon in order to prevent exposed children being dropped out from PPTCT services, subsequent mortality in absence of timely ART linkage, CPT and proper guidance on infant feeding.

Main channels for the influx of exposed children in EID remains to be either as a part of routine post-natal PPTCT services or direct in-referral from the pediatric units of the concerned healthcare setting. The latter includes migrant cases as well (Table 2).

In present study, 17 out of total 213 children (7.98\%) were reported dead at some point of time before they could reach 18 months of age. 80\% of children, dead after their testing could be done, collectively belonged to first two entry-points of EID i.e. 6 weeks or 6 months. All 7 children reported to be dead even before their testing could be done under EID were the potential entrants for 6 weeks of testing. These mortality figures further necessitate the efforts required to increase the uptake of EID services to the exposed children at the earliest in their lifetimes in order to have better ART linkage and subsequent services (Table 2).

30 out of total 213 children (14.08\%) under EID were referred in from the pediatric units based on the clinical features and symptomatology. These were the cases without their mothers being in PPTCT service loop and the children would also have missed the detection had they not been admitted to these pediatric facilities. As the name suggests, PPTCT services are comprehensively targeted towards women, children and spouses and not the individuals alone. Barring 2 cases of in-migration, ideally, rest of them should have been the part of in-referral from routine PPTCT channel, just like the $85.92 \%$ of 213 children that were part of the lineage from the mothers already availing PPTCT services. Besides, 69.4\% of the babies from regular PPTCT channel entered EID at 6-weeks-testing-protocol which ideally should have been 100\% (Table 2). Lack of knowledge in the target population of HIV sero-positive mothers regarding EID could be the major factor, especially during the initial phase service roll out. Aggressive sensitization drives through conventional healthcare delivery system as well as through networks of sero-positive people and women, is the need of hour.

Less of biohazard risk (compared to liquid samples), requirement of minimal storage facilities (since the samples are stable at room temperature for prolonged periods), easier shipping, non-requirement of highly skilled

Table 1. Distribution of exposed children getting tested under EID according to national algorithm for testing policy.

\begin{tabular}{cccc}
\hline $\begin{array}{c}\text { Time landmark at the entrance of the } \\
\text { exposed child in EID system }\end{array}$ & $\begin{array}{c}\text { Sero-positive out of all } \\
\text { children tested }\end{array}$ & $\begin{array}{c}\text { Sero-negative out of all } \\
\text { children tested }\end{array}$ & Total children tested \\
\hline New children tested at 6 weeks & 7 & 128 & 135 \\
New children tested at 6 months & 6 & 41 & 21 \\
New children tested at 12 months & 4 & 5 & 25 \\
New children tested at 18 months & 1 & $\mathbf{1 9 5}$ & $\mathbf{2 1 3}$ \\
Total & $\mathbf{1 8}$ & 213 \\
\hline
\end{tabular}

Table 2. Break-up of exposed children under EID according to their alive status and type of in-referral in EID.

\begin{tabular}{|c|c|c|c|c|c|c|c|c|c|c|c|c|}
\hline \multirow{3}{*}{$\begin{array}{l}\text { Entry point } \\
\text { in EID }\end{array}$} & \multicolumn{6}{|c|}{ Dead children while in EID } & \multicolumn{6}{|c|}{ Source-point of children's entry into EID system } \\
\hline & \multicolumn{2}{|c|}{$\begin{array}{c}\text { Death after being } \\
\text { tested }\end{array}$} & \multicolumn{2}{|c|}{$\begin{array}{c}\text { Death before being } \\
\text { tested }\end{array}$} & \multicolumn{2}{|c|}{ Total deaths } & \multicolumn{2}{|c|}{$\begin{array}{c}\text { From pediatric } \\
\text { units }\end{array}$} & \multicolumn{2}{|c|}{$\begin{array}{l}\text { Through regular } \\
\text { PPTCT channel }\end{array}$} & \multicolumn{2}{|c|}{ Total entrants } \\
\hline & $\mathbf{n}$ & $\%$ & $\mathbf{n}$ & $\%$ & $\mathbf{n}$ & $\%$ & $\mathbf{n}$ & $\%$ & $\mathbf{n}$ & $\%$ & $\mathbf{n}$ & $\%$ \\
\hline at 6 weeks & 4 & 40.00 & 7 & 100.00 & 11 & 64.71 & 8 & 27 & 127 & 69 & 135 & 63 \\
\hline at 6 months & 4 & 40.00 & 0 & 0.00 & 4 & 23.53 & 13 & 43 & 34 & 19 & 47 & 22 \\
\hline at 12 months & 2 & 20.00 & 0 & 0.00 & 2 & 11.76 & 8 & 27 & 17 & 9.3 & 25 & 12 \\
\hline at 18 months & 0 & 0.00 & 0 & 0.00 & 0 & 0.00 & 1 & 3.3 & 5 & 2.7 & 6 & 2.8 \\
\hline Total & 10 & 100.00 & 7 & 100.00 & 17 & 100.00 & 30 & 100 & 183 & 100 & 213 & 100 \\
\hline
\end{tabular}


professionals, requirement of little formal training, etc. are some of the features of DBS. PCR tests on DBS for HIV DNA demonstrate sensitivities and specificities comparable to those achieved on liquid blood [1]. Results by DBS and by confirmatory testing in our study are unequivocal hence validity in terms of $100 \%$ sensitivity and $100 \%$ specificity of DBS test at 6 weeks in our findings corroborates with the same (Table 3). Though EID in India is still a new concept in existing PPTCT infrastructure and this study might be one of the first few of those done post-implementation, validation of DBS at 6 weeks can be brought out with more evidence in future. which may help shaping country's EID program.

Various factors that may be influential towards serostatus of the child were also assessed.

For $60 \%$ of the confirmed sero-positive children, their mothers were detected HIV-sero-positive either in second or third trimester of pregnancy (Table 4). Success of PPTCT program lies in the earliest detection of pregnant woman and her subsequent linkage with service. This finding raises inevitable questions over the same. Dissemination and implementation of right messages about HIV testing of pregnant women at the antenatal facilities during their first visit is required. In $40 \%$ of the seropositive-babies, mothers were left undiagnosed for HIV due to emergency labor and were detected so post delivery. Along with universal work precautions, treating every case of emergency delivery as HIV-sero-positive delivery and emergency HIV testing of all unregistered delivery cases are the protocols that need to be implemented at grass-root level with stringent surveillance.

Mothers of $80 \%$ of confirmed sero-positive children were not on ART at the time of delivery. The reasons could be non-eligibility for ART as per their CD4 counts or a mere failure in linkage with ART services. Relative risk (RR) in terms of mother's status on ART and child's sero-positivity was 1.8 . (CI at $95 \% 0.2222$ to 16.0226 , z statistic non-significant). The idea of ART initiation regardless of CD4 counts is fast catching up. Its logic and feasibility in India needs further scientific deliberation. However, the need remains to minimize the number of eligible mothers not linked with ART to zero in order to

Table 3. Comparison of DBS results with gold-standard confirmatory test results at 18 months.

\begin{tabular}{cccc}
\hline \multirow{2}{*}{$\begin{array}{c}\text { Confirmatory } 3 \text { rapid tests } \\
\text { at } 18 \text { months }\end{array}$} & \multicolumn{2}{c}{ DBS at $\mathbf{6}$ weeks } & Total \\
\cline { 2 - 3 } & Positive & Negative & \\
\hline Positive & 2 & 0 & 2 \\
Negative & 0 & 24 & 24 \\
Total & 2 & 24 & 26 \\
\hline
\end{tabular}

Only those children included who were tested both with DBS at 6 weeks and confirmatory tests at 18 months. achieve the minimum possible maternal intra-partum viral load [5].

sdNVP is highly efficacious when given to both mother and baby (MB Pair) as per national guidelines. Provision to either of them or to none results in existence of viral load dangerous enough to contract child with HIV [7]. MB-pair was not given in $60 \%$ of such confirmed seropositive children. RR was staggering 18 and findings were statistically significant at 95\% CI 3.6943 to 87.7022 ( $\mathrm{p}<0.0003$ ). Jain et al. reported significantly less HIV positivity in children receiving ARV prophylaxis in the form of sdNVP than those who did not [4]. Studies from other countries such as Botswana [8] and Tanzania [9] also show low prevalence in children receiving the Nevirapine at the time of birth. This difference emphasizes the need for strengthening the strategy of giving ARVs for MTCT [4]. NVP alone may have resistance-issues. Multidrug regime including NVP and Zydovudine which is already principally accepted for better results at national level, should be a ground reality soon across the country.

National guidelines advocates opting for elective cesarean section of sero-positive children in order to minimize HIV transmitting possibilities, though the risk is equal for both vaginal and cesarean modes when mother is on ART [7]. Cesarean section (CS) is believed to decrease transmission of HIV as documented in a Kenyan study [10]. In our study, for $60 \%$ of confirmed seropositive children, mode of delivery was vaginal and the deliveries were handled in emergency. However, the difference was statistically insignificant but it corroborates with the findings of Jain et al. in Gujarat [4].

Current national guidelines suggests exclusive breastfeeding (EBF) in absence of exclusive top feeding is the preferred infant feeding method, other options being wet nursing and expressed milk feeding. In any case, no two milks should be mixed. If EBF is opted, it is to be continued till 6 months followed by weaning, transition spread over a period of one week. In developing country like India with majority of the sero-positive women belonging to lower socio-economic strata, EBF is generally the preferred way meeting the AFASS criteria [7]. confirmatory testing is done when the baby is off mother's breast for at least 6 weeks. History of exclusive breastfeeding was reported in $60 \%$ of confirmed sero-positive children while in only $20 \%$ of sero-negative babies. In rest, improper feeding practices were followed (z statistic non-significant). National guidelines on infant feeding have changed from time to time and it may require more deliberation.

System for earliest detection of exposed child for HIV and subsequent ART linkage is in place in the country but it is still in the initial phase. Validity of the earliest testing under EID at 6 weeks is satisfactory compared to 
Table 4. Correlation of sero-status of exposed children undergoing confirmatory test with various peri-partum factors.

\begin{tabular}{|c|c|c|c|c|c|}
\hline \multirow{2}{*}{\multicolumn{2}{|c|}{ Determinants for children tested with confirmatory rapid tests at 18 months }} & \multicolumn{2}{|c|}{ Confirmed sero-positives } & \multicolumn{2}{|c|}{ Confirmed sero-negatives } \\
\hline & & $\mathbf{n}$ & $\%$ & $\mathbf{n}$ & $\%$ \\
\hline \multirow{5}{*}{$\begin{array}{l}\text { Time of detection of mother's } \\
\text { sero-status for HIV }\end{array}$} & 1st trimester & 0 & 0 & 9 & 12.3 \\
\hline & 2nd trimester & 2 & 40 & 26 & 35.6 \\
\hline & 3rd trimester & 1 & 20 & 31 & 42.5 \\
\hline & $\begin{array}{l}\text { Mother was not detected during pregnancy, } \\
\text { identified post-delivery }\end{array}$ & 2 & 40 & 7 & 9.6 \\
\hline & Total & 5 & 100.00 & 73 & 100.00 \\
\hline \multirow{3}{*}{$\begin{array}{l}\text { History of mother on ART at the } \\
\text { time of delivery }\end{array}$} & Mother was not ART & 4 & 80.00 & 49 & 67.1 \\
\hline & Mother was on ART & 1 & 20.00 & 24 & 32.9 \\
\hline & Total & 5 & 100.00 & 73 & 100.00 \\
\hline \multirow{3}{*}{$\begin{array}{l}\text { History of sdNVP in the form } \\
\text { of mother-baby pair }\end{array}$} & Mother-baby pair of sdNVP was not given & 3 & 60.0 & 3 & 4.1 \\
\hline & sdNVP was given to both mother and baby & 2 & 40.0 & 70 & 95.9 \\
\hline & Total & 5 & 100.0 & 73 & 100.0 \\
\hline \multirow{3}{*}{ Mode of delivery } & Mode of delivery-vaginal & 3 & 60.0 & 48 & 65.8 \\
\hline & Mode of delivery-cesarean section & 2 & 40.0 & 25 & 34.3 \\
\hline & Total & 5 & 100.0 & 73 & 100.0 \\
\hline \multirow{3}{*}{ Type of delivery } & Emergency delivery & 3 & 60.0 & 46 & 63.0 \\
\hline & Planned delivery & 2 & 40.0 & 27 & 37.0 \\
\hline & Total & 5 & 100.0 & 73 & 100.0 \\
\hline \multirow{3}{*}{ Feeding history } & $\begin{array}{l}\text { Other type of feeding (top, mixed, replacement, } \\
\text { any other mode) }\end{array}$ & 2 & 40.0 & 58 & 79.5 \\
\hline & History of exclusive breast feeding by the mother & 3 & 60.0 & 15 & 20.6 \\
\hline & Total & 5 & 100.0 & 73 & 100.0 \\
\hline
\end{tabular}

All exposed children undergoing confirmatory test at 18 months regardless of type of in-referral or current alive status are included.

gold standard rapid testing at 18 months. During earlier times, linkage of exposed child with ART services would have been possible only after confirmatory testing at 18 months. However, significant number of exposed children can be saved from dying in infancy and be provided with prolonged and quality life with timely HIV detection and ART linkage. Considering the huge population burden of India, geographical vastness and HIV/AIDS being urban-concentrated epidemic; uniform and concerted efforts especially in city areas are required to increase EID service uptake. Earliest detection of pregnant woman, HIV testing for emergency deliveries, intrapartum sdNVP to both mother and baby and linkage of eligible mothers with ART still remain cornerstone of prevention of vertical HIV transmission.

\section{LIMITATIONS}

EID was started in Ahmedabad since 18 months at the time of the study and present study was based on the observations of only 213 children enrolled in the system thus far. The PPTCT services are ongoing and further validation of data with more evidences from such cohorts may help immensely in the national programmatic ratifications.

\section{ACKNOWLEDGEMENTS}

We thank the PPTCT counselors of AMC-ACS, Ms. Shilpa Patel, Ms. Nimita Patel, Ms. Kusum Vyas and Ms. Heena Bhanderi for their support and inputs towards the study. We also thank all the women participants and their children for making this study possible.

\section{REFERENCES}

[1] Sherman, G.G., Stevens, G., Jones, Stephanie, A., Horsfield, P. and Stevens, W.S. (2005) Dried blood spots improve access to HIV diagnosis and care for infants in 
low-resource settings. Journal of Acquired Immune Deficiency Syndromes, 38, 615-617.

doi:10.1097/01.qai.0000143604.71857.5d

[2] Creek, T.L., Sherman, G.G., Nkengasong, J., Lu, L., Finkbeiner, T., Fowler, M.G., et al. (2007) Infant human immunodeficiency virus diagnosis in resource-limited settings: Issues, technologies, and country experiences. American Journal of Obstetrics \& Gynecology, 197, S64S71. doi:10.1016/j.ajog.2007.03.002

[3] Stevens, W., Sherman, G., Downing, R., Parsons, L.M., Ou, C.Y., Crowley, S., et al. (2008) Role of the laboratory in ensuring global access to ARV treatment for HIVinfected children: Consensus statement on the performance of laboratory assays for early infant diagnosis. Open AIDS Journal, 2, 17-25. doi:10.2174/1874613600802010017

[4] Jain, K., Mahajan, R., Shevkani, M. and Gupta, P.K. (2011) Early infant diagnosis: A new tool of HIV diagnosis in children. Indian Journal of Community Medicine, 36, 139-142.

[5] National AIDS Control Organization (2006) Guidelines for HIV care and treatment in infants and children. http://www.nacoonline.org/About_NACO/Policy_Guide
lines/Policies_Guidelines_Table_for_download/

[6] Sentinel Surveillance Report.

[7] National AIDS Control Organization: Guidelines for the prevention of mother to child transmission of HIV.

[8] Creek, T. (2008) Early diagnosis of human immunodeficiency virus in infants using polymerase chain reaction on dried blood spots in Botswana's national program for prevention of mother-to-child transmission. Pediatric Infectious Disease Journal, 27, 22-26. doi:10.1097/INF.0b013e3181469050

[9] Nuwagaba-Biribonwoha, H., Werq-Semo, B., Abdallah, A., Cunningham, A., Gamaliel, J.G., Mtunga, S., et al. (2010) Introducing a multi-site program for early diagnosis of HIV infection among HIV-exposed infants in Tanzania. BMC Pediatrics, 10, 44. doi:10.1186/1471-2431-10-44

[10] Pathfinder International/ Kenya's Prevention of Mother to Child Transmission Project Early Infant Diagnosis of HIV through Dried Blood Spot Testing (2007) http://www.pathfind.org/site/DocServer/Kenya_EID.pdf? $\underline{\text { docID }=10201}$

\author{
Abbreviations \\ PMTCT $=$ Prevention from Mother to Child Transmission \\ PPTCT $=$ Prevention from Parent to Child Transmission \\ NACP III = National AIDS Control Program Phase 3 \\ EID = Early Infant Diagnosis \\ DBS $=$ Dried Blood Spot \\ WB $=$ Whole Blood Collection \\ DNA-PCR = DNA-Polymerase Chain Reaction
}

\author{
ARV $=$ Antiretroviral \\ ART = Antiretroviral Therapy \\ sdNVP = Single Dose Nevirapine \\ $\mathrm{RR}=$ Relative Risk \\ $\mathrm{CI}=$ Confidence Interval \\ AMC $=$ Ahmedabad Municipal Corporation \\ NACO = National AIDS Control Organization \\ CPT $=$ Cotrimoxazole Prophylaxis Therapy
}

